\title{
Picroside II attenuates ischemia/reperfusion testicular injury by alleviating oxidative stress and apoptosis through reducing nitric oxide synthesis ${ }^{1}$
}

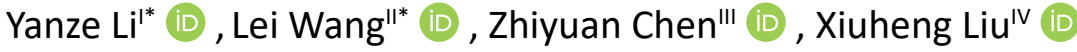

' Master, Department of Urology, Renmin Hospital of Wuhan University, Wuhan, Hubei, P.R. China. Design of the study, technical procedures, interpretation of data, statistical analysis, manuscript preparation.

" MD, Department of Urology, Renmin Hospital of Wuhan University, Wuhan, Hubei, P.R. China. Design of the study, interpretation of data.

I"Associate Professor, Department of Urology, Renmin Hospital of Wuhan University, Wuhan, Hubei, P.R. China. Conception and design of the study, critical revision.

IV Full Professor, Department of Urology, Renmin Hospital of Wuhan University, Wuhan, Hubei, P.R. China. Conception and design of the study, critical revision, final approval.

*The authors contributed equally to this work

\begin{abstract}
Purpose: To investigate the effect of Picroside II on testicular ischemia and reperfusion (I/R) injury and the underlying mechanism.

Methods: Sprague-Dawley rats were randomly divided into 4 groups: sham operated group (Sham), Sham with Picroside II treatment group (Sham+ Pic II), I/R group (I/R) and I/R with Picroside II treatment group (I/R+ Pic II). I/R model was established by rotating the left testis $720^{\circ}$ in a clock-wise direction for 4 hours. The histopathologic and spermatogenetic evaluation was performed. The apoptosis changes and the levels of HO-1 (heme oxygenase-1), MPO (myeloperoxidase), NOX (NADPH oxidase), SOD (superoxide dismutase), XO (xanthine oxidase) and NOS (nitric oxide synthase) were measured.

Results: The seminiferous tubules were damaged in I/R rats, but Picroside II alleviated the changes induced by I/R. The increased level of apoptosis was decreased by Picroside II $(P=0.01,9.05 \pm 0.35$ vs. 4.85 \pm 0.25$)$. The activities of HO-1, MPO, NOX, XO and MDA content were increased and the SOD activity was decreased in $I / R(P<0.05)$ and could be reversed by Picroside II $(P=0.03,405.5 \pm 7.5$ vs. $304 \pm 17 \mathrm{U} / \mathrm{mgprot} ; \mathrm{P}=0.02,0.99 \pm 0.05$ vs. $0.52 \pm 0.04$ mgprot; $P=0.01,260 \pm 7$ vs. $189 \pm 2$ mgprot; $P=0.04$, $10.95 \pm 0.55$ vs. $8.75 \pm 0.35 \mathrm{U} / \mathrm{mgprot} ; \mathrm{P}=0.045,6.8 \pm 0.7$ vs. $3.75 \pm 0.35$ mgprot; $\mathrm{P}=0.04,44.5 \pm 3.5$ vs. $57.5 \pm 3.5 \mathrm{mgprot})$. Western blot showed that the expression of iNOS, nNOS and eNOS were increased in I/R $(P<0.05)$; however, they were decreased after Picroside II treatment $(P<0.05)$.
\end{abstract}

Conclusion: Picroside II attenuated testicular I/R injury in rats mainly through suppressing apoptosis and oxidative stress through reduction of nitric oxide synthesis.

Key words: Picroside. Ischemia. Reperfusion. Oxidative Stress. Apoptosis. Testis. Rats. 


\section{- Introduction}

Testis torsion, a rotating of the spermatic cord and its contents, is a urology emergency. Annual incidence of spermatic cord torsion is 4.5 in 100.000 males $1-25$ years of age ${ }^{1}$. If treated within 6 hours of the presenting pain, there is a good chance of saving the affected testicle, as $90 \%-100 \%$ testicles will be saved. If treated within $6 \mathrm{~h}-12 \mathrm{~h}$, about 20\%-50\% testicles will be saved and if treated within $12 \mathrm{~h}-24 \mathrm{~h}$, only $0 \%-10 \%$ testicles will be saved $^{2}$. The degree of testis damage is dependent on the torsion degree and duration ${ }^{3}$. It was reported that survival rates of surgical detorsion was ranging from $42 \%$ to $88 \%$, but the testicular function was unpredictable.

The pathophysiology of testis depended on ischemia/reperfusion (I/R) injury. And overgeneration of reactive oxygen species (ROS) plays a crucial role in I/R injury, causing the damage of testis spermatogenesis ${ }^{4}$. During reperfusion, ROS stimulated the generation and recruitment of inflammatory cytokines (such as interleukin-1 $\beta$ (IL-1 $\beta$ ) and tumor necrosis factor- $\alpha$ (TNF- $\alpha$ ), leading to testicular atrophy, germ cell apoptosis and destruction of spermatogenesis ${ }^{5,6}$.

Picroside II is a flavonoid compound extracted from a traditional Chinese herbal medicine, Picrorhiza scrophulariiflora pennell. Previously, it has been demonstrated that Picroside II possesses many effects, such as anti-oxidative stress, anti-apoptosis and antiinflammation effects ${ }^{7,8}$. Picroside II has been applied to protect against I/R injury in many organs, including the brain, kidney and so on ${ }^{9-11}$. However, its effect on testis $\mathrm{I} / \mathrm{R}$ has not been reported. In this study, we aimed to investigate the effects of Picroside II on testicular I/R injury and the underlying mechanism.

\section{- Methods}

All 24 adult male Sprague-Dawley rats (6-8 weeks, 220-250 g) were from the Center of Experimental Animals in Medical College, Wuhan University. The committee of experimental animals of Wuhan University approved this project, and the procedures carried out according to the routine animal-care guidelines.

All rats were housed at $20-24^{\circ} \mathrm{C}$ with $12 \mathrm{~h}$ light- $12 \mathrm{~h}$ dark cycle and supplied with standard rat chow and water.

\section{$I / R$ procedures}

The rats in each group were anesthetized with pentobarbital (45 $\mathrm{mg} / \mathrm{kg})$ and placed on a homeothermic table to maintain core body temperature at $37{ }^{\circ} \mathrm{C}$. All operations were performed under sterile conditions. Briefly, after anesthesia, a vertical paramedian incision of the scrotum was made and then unilateral testicular torsion was performed by rotating the left testis $720^{\circ}$ in a clockwise direction and fixed for 4 hours. At the end of 4 hours, the detorsion of the testis was performed after anesthesia and the testis was placed back into the scrotum ${ }^{12}$.

\section{Experimental protocol}

All rats were randomly divided into four groups, each with six rats. Sham group was subjected to all operative procedures, except testis torsion and detorsion. Sham+ Pic II (Picroside II) group was the same as Sham group, together with administration of Picroside II (10 mg/kg). In I/R group, rats undergone 4 hours of testis torsion and 4 hours of detorsion. I/R+Pic II group was administrated with Picroside II (10 mg/kg) 30 minutes before testis detorsion, and the other procedures were the same with I/R group.

\section{Preservation of testis}

The testis was removed under fully maintained anesthesia at the end of detorsion. After removal, the testis was fixed in $10 \%$ phosphate-buffered formalin, and stored at $-80^{\circ} \mathrm{C}$ for the following experiments.

\section{Intervention study}

Picroside II (CAS No: 39012-20-9, purity >98\%, molecular formula $\mathrm{C} 23 \mathrm{H} 28013)$ was purchased from Tianjin Kuiqing Medical Technology Co. Ltd. We diluted it into $10 \mathrm{~g} / \mathrm{L}$ solution with $1 \mathrm{~mol} / \mathrm{L}$ PBS. Picroside II $(10 \mathrm{mg} / \mathrm{kg}) 250 \mu \mathrm{L}$ was administrated via tail vein with a micro-syringe in Sham+ Pic II group and I/R+ Pic II group. And rats in $\mathrm{I} / \mathrm{R}$ group and Sham group were injected $1 \mathrm{~mol} / \mathrm{L}$ PBS $250 \mathrm{uL}$ simultaneously.

\section{Hypothesis}

After torsion/detorsion, testis structure will be destroyed, and the level of apoptosis will increase, and the level of oxidative stress will also ascent, and meanwhile the expression of NOS will grow. However, after the administration of Picroside II, all the changes will be reversed.

\section{Primary outcome measurements}

Histologic examinations of testis tissues to observe the morphologic changes; TUNEL to learn the level of apoptosis; detection of some markers (like HO-1, SOD, NOX, etc.) to find the changes in oxidative stress; western blotting to test the expressions of nitric oxide synthesis. 


\section{Secondary outcome measurement}

The mean seminiferous tubule diameter (MSTD) and TUNEL index.

\section{Histologic examinations}

The testis was fixed in 10\% phosphate -buffered formalin, and then embedded with paraffin and sectioned at $4-\mu \mathrm{m}$ thick. The sections were deparaffinized and hydrated, and stained with hematoxylin and eosin (H\&E). Morphologic evaluations were performed by an experienced pathologist who didn't know the protocol. The mean seminiferous tubule diameter (MSTD) was measured and the Johnsen scores were evaluated according to Johnsen's Tubular Biopsy Scores (JTBS) and Table $1^{13}$.

Table 1 - Histological criteria and the modified Johnsen score system for assessment of spermatogenesis.

\begin{tabular}{lc} 
Score & Histologic properties \\
\hline 10 & Full spermatogenesis \\
9 & Slightly impaired spermatogenesis, many late spermatids, disorganized epithelium \\
8 & Less than five spermatozoa per tubule, few late spermatids \\
7 & No spermatozoa, no late spermatids, many early spermatids \\
6 & No spermatozoa, no late spermatids, few early spermatids \\
5 & No spermatozoa or spermatids, many spermatocytes \\
4 & No spermatozoa or spermatids, few spermatocytes \\
3 & Spermatogonia only \\
2 & No germinal cells, Sertoli cells only \\
1 & No seminiferous epithelium \\
\hline
\end{tabular}

\section{TUNEL assay}

Apoptotic level on paraffin sections of the testis was assessed by TUNEL (Terminal-deoxynucleotidylTransferase-mediated dUTP Nick End Labeling) with a commercial kit (Boehringer Mannheim, Mannheim, Germany). Control slides were incubated with $50 \mu \mathrm{L}$ Label solution and stained with $0.05 \%$ diaminobenzidine for $10 \mathrm{~min}$. And then, we counterstained sections with Mayer's haematoxylin and observed them under a Nikon Eclipse 50i microscope at 400x magnification. Positive cells were marked by brown staining. The average number of TUNEL-positive cells per tubule was calculated in 10 random fields.

\section{Heme oxygenase-1 activity}

Briefly, testis tissue was added to a reaction mixture. The reaction was performed at $37^{\circ} \mathrm{C}$ in the dark condition for 1 hour and then it was terminated by the addition of $1 \mathrm{~mL}$ of chloroform. The extracted bilirubin was calculated by the difference in absorbance between the wavelengths 464 and $530 \mathrm{~nm}$.

\section{Nicotinamide adenine dinucleotide phosphate oxidase activity}

Lucigenin-enhanced chemiluminescence was used to measure nicotinamide adenine dinucleotide phosphate (NADPH) oxidase activity in testis, according to the method described previously ${ }^{14}$.

\section{Measurement of Myeloperoxidase Activity}

Activity of testicular myeloperoxidase (MPO), an enzyme that is found predominantly in the azurophilic granules of polymorphonuclear leukocytes, was measured by the method described previously ${ }^{15}$.

\section{Superoxide dismutase (SOD) and malondialdehyde (MDA) measurement}

Commercial kits were used in accordance with the manufacturer's instructions (Nanjing Jiancheng Co., China) to measure SOD activity (xanthineoxidase method) and MDA concentration (thiobarbituric acid method). 


\section{Assay for xanthine oxidase activity}

Xanthine Oxidase Activity was measured by the method described previously ${ }^{15}$.

\section{Western Blot analysis}

Total proteins were separated, and quantified by Bicinchoninic acid method. Then, equivalent weights of protein $(40 \mu \mathrm{g} /$ lane) were separated on $10 \%$ SDS-PAGE gels and transferred to nitrocellulose membrane. 5\% non-fat milk was used to block the membranes in TBST buffer. Then the membranes were incubated with primary antibodies against eNOS, nNOS, and iNOS (all obtained from Santa Cruz) at $4^{\circ} \mathrm{C}$ using slow rocking. Next, after washing twice with TBST, the membranes were blotted with secondary antibody, which was conjugated with horseradish peroxidase at 1:5000 dilution. Distinct bands were emphasized by using a chemiluminescence detection kit.

\section{Statistical analysis}

The means of sample sizes were calculated in each group and data were presented as mean \pm SEM. Student $t$ and One-way ANOVA test with Bonferroni adjustment was used for comparing normally distributed data.
Then the means of the different groups were compared by using one-way ANOVA and Student-NewmanKeuls test. When $P<0.05$, differences were considered statistically significant.

\section{- Results}

\section{Effect of Picroside II on testicular damage induced by $I / R$ in rats}

The rats in the Sham group and Sham + Pic Il group both showed almost normal testis structure (Fig. 1A, B). And the rats in the I/R group showed destruction of tubular epithelium, necrosis, debris formation, congestion and atrophy (Fig. 1C). However, in I/R+ Pic II group, the testis structure was obviously improved (Fig. 1D).

According to Johnsen scoring system, the spermatogenesis in I/R group was reduced in comparison to the Sham and Sham+ Pic II group. Whereas, Picroside II treatment could reverse the reduction of spermatogenesis induced by testicular I/R (Fig. 1E). In addition, MSTD results showed that the Picroside II treatment had a larger MSTD than the I/R group (Fig. 1F).
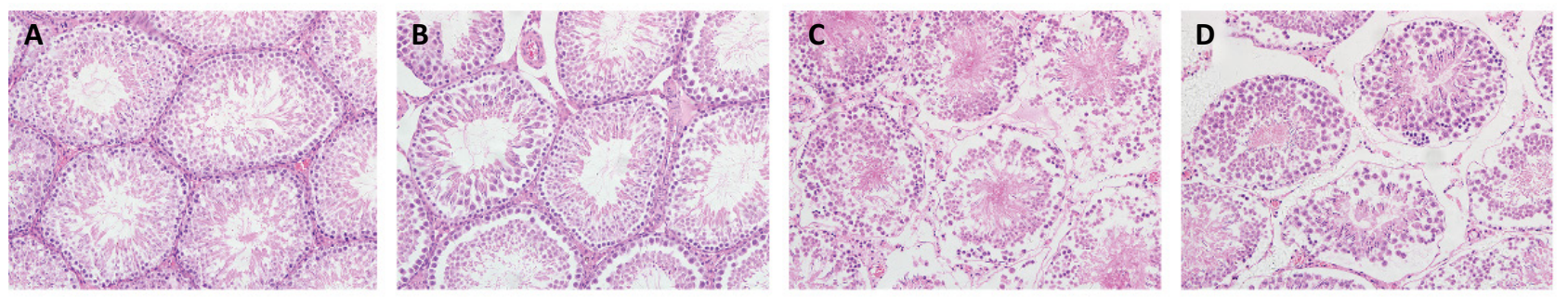

$\mathbf{E}$

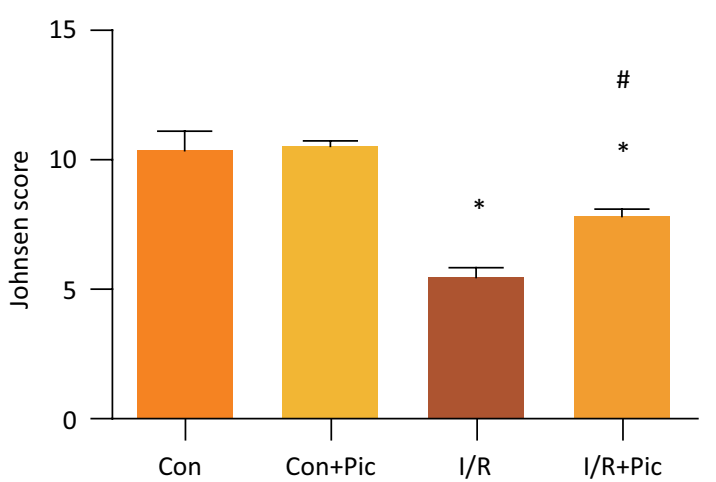

$\mathbf{F}$

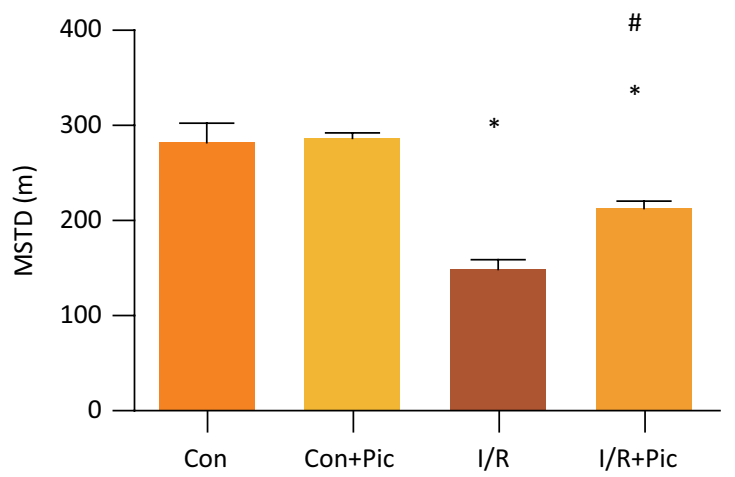

Figure 1 - Effect of Picroside II treatment on testicular damage induced by ischemia/reperfusion (I/R) in rats. Paraffin-embedded testes sections were stained with hematoxylin and eosin. histopathological evaluation: (A) sham group, (B) sham+ Pic II group, (C) I/R group, (D) I/R +Pic II group. (E, F) Johnsen scores and quantification of mean seminiferous tubular diameter (MSTD) for each group. Values are means \pm standard deviation, $n=6$ in each group. ${ }^{*} P<0.05$ vs. control group. $\# P<0.05$ vs. I/R group. 


\section{Effect of Picroside II treatment on testicular apoptosis induced by $I / R$ in rats}

TUNEL assay was used to investigate the apoptosis level induced by $\mathrm{I} / \mathrm{R}^{16}$. Contrasted with Sham group and Sham+ Pic II group (Fig. 2 A,B), it had more TUNEL positive cells in I/R group (Fig. 2C). However, in I/R+ Pic II group, Picroside II treatment could inhibit cell apoptosis induced by testicular I/R (Fig. 2D). Apoptosis index was the quantification of TUNEL assay (Fig. 2E).
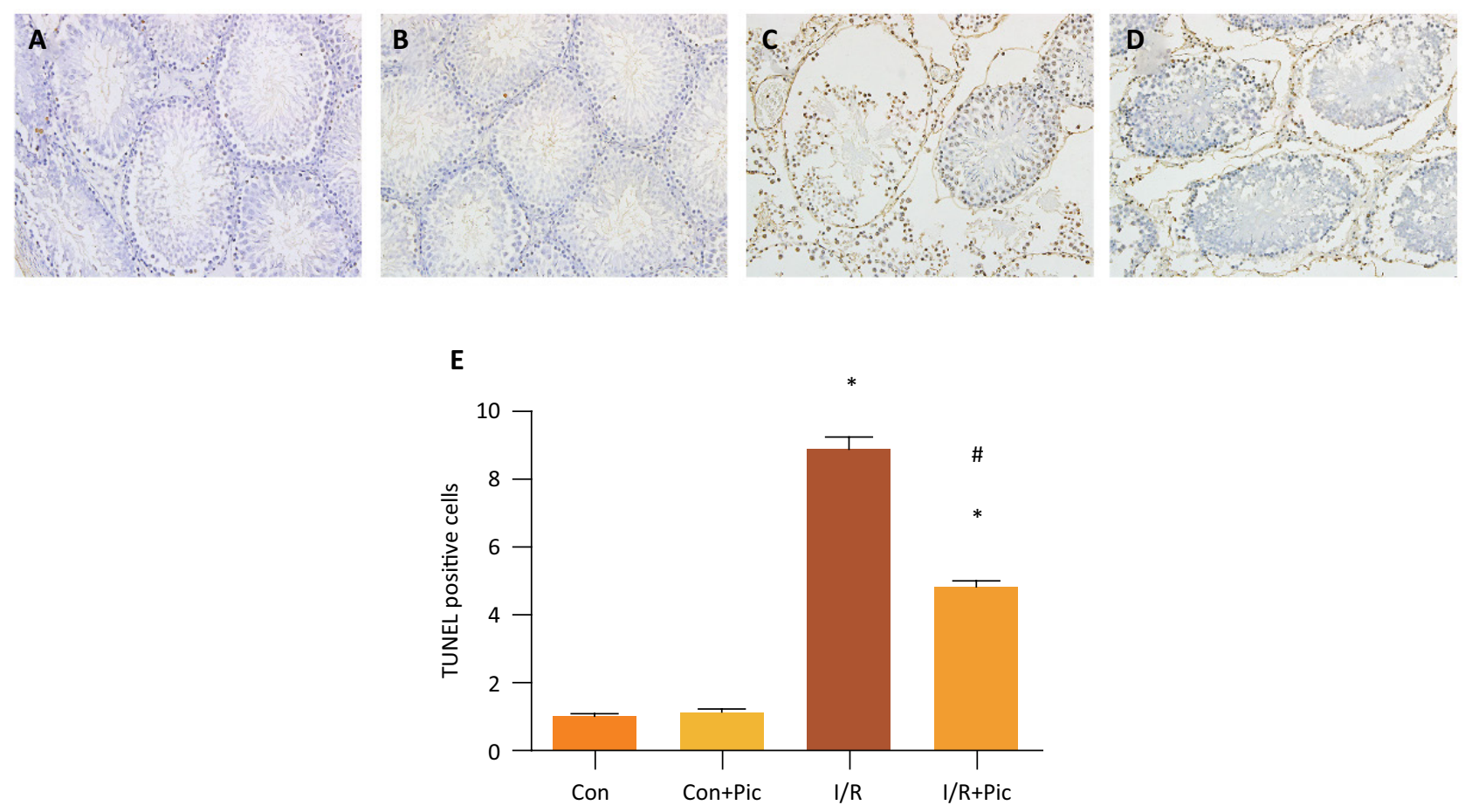

Figure 2 - Effect of Picroside II treatment on testicular cells apoptosis induced by ischemia/reperfusion(I/R) injury in rats. (A, B) Sham group and Sham+ Pic II group with a few positive cells. (C) I/R group with a high level of positive cells. (D) I/R+ Pic II group with a lower level of positive cells than I/R group. (E) apoptosis index of each group. Values are means \pm standard deviation, $n=6$ in each group. ${ }^{*} \mathrm{P}<0.05$ vs. control group. $\# \mathrm{P}<0.05$ vs. I/R group.

\section{Effect of Picroside II treatment on} testicular oxidative stress (OS) induced by ischemia/reperfusion $(I / R)$ injury in rats

The activities of HO-1, NOX, MPO, SOD, XO and content of MDA were detected to reflect rats testicular OS level induced by I/R. As the results showed, all of them had no difference between Sham group and Sham+ Pic II group (Table 2). However, the activities of HO-1, NOX, MPO, XO and content of MDA were downregulated in I/R group, when compared with Sham group and Sham+ Pic II group. Moreover, the treatment of Picroside II could improve the activities of HO-1, NOX, MPO, XO and content of MDA.
Besides, we also found that Picroside II could reverse the decreased SOD activity induced by testicular $I / R$ injury. All these results indicated that Picroside II could improve the oxidative stress induced by $I / R$ in testis.

The effect of Picroside II treatment on testicular oxidative stress (OS) induced by ischemia/reperfusion $(\mathrm{I} / \mathrm{R})$ injury in rats was evaluated. After 4-hour detorsion, testes were removed for determination of activities of heme oxygenase-1 (HO-1), NADPH oxidase (NOX), myeloperoxidase (MPO), superoxide dismutase (SOD), xanthine oxidase (XO) and malondialdehyde (MDA) content. Values are means \pm standard deviation, $n=6$ in each group (Table 2 ). 
Table 2 - Evaluation of oxidative stress markers for each group.

\begin{tabular}{|c|c|c|c|c|c|}
\hline & Sham $(n=6)$ & Sham+PicII $(n=6)$ & $I / R(n=6)$ & I/R+PicII $(n=6)$ & P (ANOVA) \\
\hline HO-1 activity (U/mgprot) & $212 \pm 11$ & $221 \pm 4$ & $406 \pm 8$ & $304 \pm 17$ & $<0.05$ \\
\hline NOX activity (U/mgprot) & $107 \pm 6$ & $108 \pm 1$ & $260 \pm 7$ & $189 \pm 2$ & $<0.05$ \\
\hline MPO activity (U/mgprot) & $0.18 \pm 0.06$ & $0.16 \pm 0.05$ & $0.99 \pm 0.05$ & $0.52 \pm 0.04$ & $<0.05$ \\
\hline SOD(U/mgprot) & $74.5 \pm 4.5$ & $77.5 \pm 1.5$ & $44.5 \pm 3.5$ & $57.5 \pm 3.5$ & $<0.05$ \\
\hline XO activity (U/mgprot) & $6.00 \pm 0.10$ & $5.95 \pm 0.05$ & $10.95 \pm 0.55$ & $8.75 \pm 0.35$ & $<0.05$ \\
\hline MDA (nmol/mgprot) & $2.3 \pm 0.3$ & $2.3 \pm 0.2$ & $6.8 \pm 0.7$ & $3.8 \pm 0.4$ & $<0.05$ \\
\hline
\end{tabular}

\section{Effect of Picroside II treatment on testicular nitric} oxide synthesis induced by ischemia/reperfusion $(I / R)$ injury in rats

Nitric oxide synthase (NOS), including three isoenzymes (nNOS, eNOS, iNOS), is the rate-limiting enzyme in the synthesis of nitric oxide, and its activity changes directly regulate the formation of nitric oxide and its biological effects ${ }^{17}$. Western blot results showed that the expression of nNOS, eNOS and iNOS had no difference between Sham group and Sham+ Pic II group. However, I/R could induce the expression of them and Picroside II treatment could reverse the increased expression induced by I/R (Fig. 3 A-D).
A

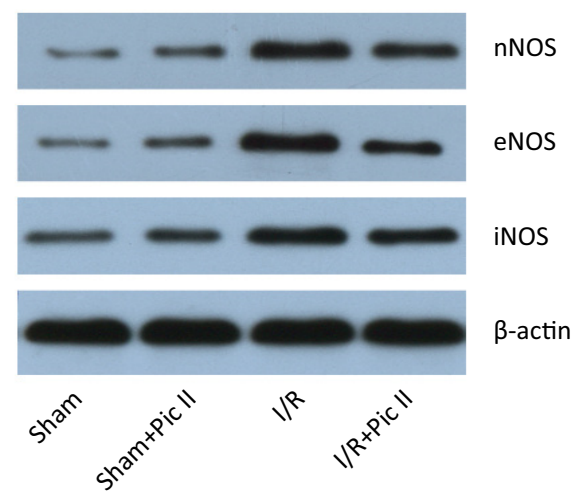

C

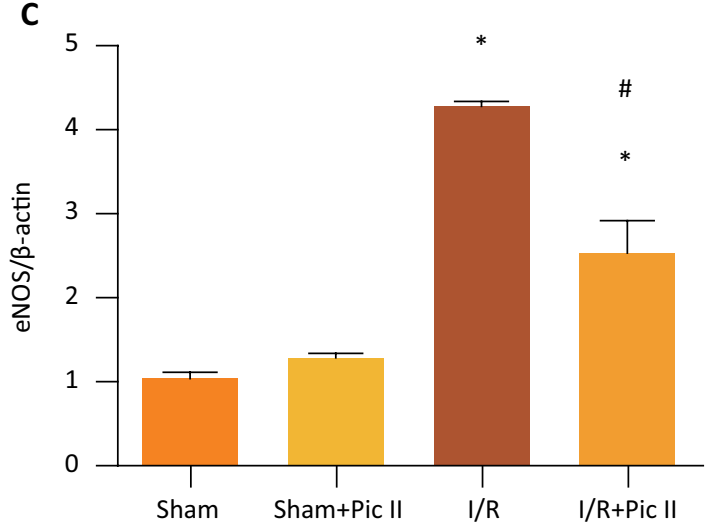

B

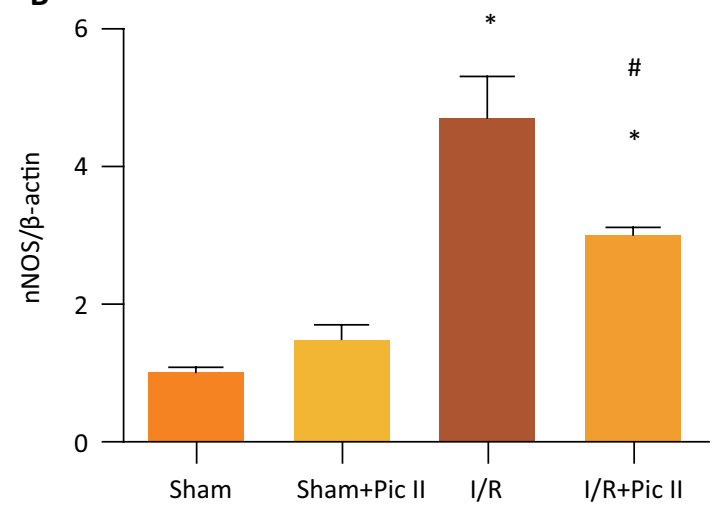

D

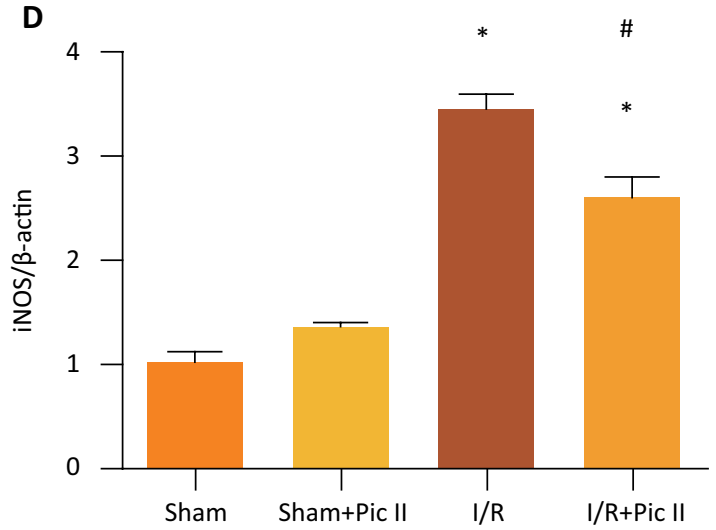

Figure 3 - Effect of Picroside II treatment on testicular nitric oxide (NO) synthesis induced by ischemia/reperfusion (I/R) injury in rats. After 4-hour detorsion, Testis were removed for western blotting (A) to analyze protein expression of nNOS (B), eNOS, (C) iNOS (D). Values are means \pm standard deviation, $n=6$ in each group. $* \mathrm{P}<0.05$ vs. control group. \#P $<0.05$ vs. I/R group. 


\section{- Discussion}

Testis torsion is an emergency in the urology department, usually in childhood or adolescence. Successful surgery for detorsion is necessary, but there are still $40 \%-80 \%$ patients with poor prognosis, including testicular atrophy and infertility. I/R injury in torsion/ detorsion is the direct and complex damage, including the significant increase of reactive oxygen radicals (ROS) and reactive nitrogen species (RNS) ${ }^{18}$. In our study, we performed testis torsion/detorsion as the model of testicular I/R injury.

Picroside II, a Chinese herb extract, has demonstrated pharmacological effects, such as antioxidant, anti-apoptotic, anti-inflammatory, anticarcinogenic, neuroprotective, hepatoprotective, anticholestatic effects, and immune modulating activities. Its effects are much stronger than those of other types of flavonoid. Due to its effects, Picroside II has proved to be effective on protecting against I/R injury, including heart, liver and kidney ${ }^{9-11}$. Although the effect of some other drugs hasbeen studied $t$ on testicular $I / R$, there aren't many researches applying Chinese herb extracts, particularly Picroside $\mathrm{II}^{19-22}$.

It has been reported that testicular injury consists of structure destruction in morphology and spermatogenesis dysfunction. Previous studies showed that in $1 / R$ injury, testicular structure might change obviously, including testis weight decrease, atrophy, destruction of tubular epithelium, necrosis, debris formation and congestion ${ }^{23,24}$. In our study, the results showed the same. It is known that germ cells are the most sensitive to I/R injury, especially to lipid peroxidation, because there is a rich content of polyunsaturated fatty acids in their plasma membrane ${ }^{25,26}$. With the testis structure destruction, the function of spermatogenesis was also impaired, which was demonstrated based on Johnsen scoring system in our study ${ }^{27}$. However, the treatment of Picroside II could ameliorate testicular I/R injury, both in structure and function.

A previous study showed that testis I/R injury could produce large amount of ROS and inflammatory factors and enhance the spermatogenic cells apoptosis, leading to infertility ${ }^{28-30}$. In our study, we applied TUNEL assay to reveal the level of apoptosis induced by I/R. Our results were consistent with this previous study, as testis cells apoptosis level was increased after I/R; however, with Picroside II treatment, the apoptosis induced by I/R could be reduced.

I/R could cause oxidative stress by producing over production of reactive oxygen species (ROS), then leading to the destruction of lipids, proteins and DNA ${ }^{31,32}$. Based on this, we detected some markers to evaluate the changes of oxidative stress.
Heme oxygenase-1(HO-1) is a rate-limiting enzyme for heme degradation, and the degradation products have antioxidative function ${ }^{33}$. 'It's been reported that HO-1 level increases in oxidative stress reaction ${ }^{34}$. NADPH oxidase (NOX) is a major source of ROS in a number of non-phagocytic cells, and its level changes indicate the variation of $\mathrm{ROS}^{35}$. Myeloperoxidase, MPO, is an important iron-containing lysosome. It's a functional marker and activation marker of neutrophils, and its level and activity changes represent the function and activity status of neutrophilic polymorphonuclear leukocytes $(\mathrm{PMN})^{36}$. In our study, their level was increased after I/R; however, the treatment of Picroside II could significantly decrease the elevated level of them induced by $\mathrm{I} / \mathrm{R}$.

As we all know, superoxide dismutase (SOD) is an oxygen free radical scavenger, which is widely found in various tissues of organisms and can scavenge free radicals. And Malondialdehyde (MDA) is one of the most important products of membrane lipid peroxidation, which could be used to reflect the degree of membrane lipid peroxidation. Previous studies have shown that in testicular I/R injury, SOD activity is reduced and the content of MDA is increased, aggravating the damage of oxygen free radicals in testicular tissue ${ }^{37}$. Our results were consistent with previous results. SOD activity decreased and MDA content increased after I/R. However, treatment of Picroside II could obviously reverse the changed level induced by I/R. So, we consider that Picroside II could alleviate oxidative stress induced by testicular I/R injury.

Nitric oxide synthase (NOs) is the rate-limiting enzyme in the synthesis of nitric oxide, which is involved in the regulation of NO synthesis. It includes three different isoforms: endothelial NOS (eNOS), neuronal NOS (nNOS), and inducible NOS (iNOS). Our results showed that iNOS, eNOS, nNOS levels were increased after I/R injury and with the treatment of Picroside II, their upregulated levels were decreased. Therefore, we considered that Picroside II reduced NO synthesis to protect testis from I/R injury.

Comparing with the previous studies which used different kinds of treatments ${ }^{19-22}$, like nifedipine, urapidil and apocynin, we all got approximately the same results in the changes of testicular structures, the level of apoptosis and the level of some oxidative stress markers, including MDA and SOD. Nonetheless, there are also some differences between our studies. We detected more markers like HO-1, MPO, XO, and so on, and some of the previous studies detected some other markers like GPX, GSH, CAT, and so on. So, in a future study, we could use other makers for reference to do more research. Another thing that we observed is 
that we also analyzed the expression of three kinds of NOS respectively, indicating the role of NO synthesis in the testicular I/R injury ${ }^{19-22}$.

Overall, we found that Picroside II could protect against testicular I/R injury and might be a potential therapeutic agent in the future. But there were still some limitations in our study. The first one is that the sample size was calculated, as we referred to some articles and used "6 rats" in each group. But the results were measured by calculating the mean rats in each group. The second one is that, as some reports have showed that NO has double-edged effects in I/R injury, which is dependent on the tissue, site, source, and environment, in our next research, we will investigate more about $\mathrm{NO}$ effects in testis $I / R$ injury. Finally, the third one was that there was no experiment in vitro; so, next time we will need to conduct an in vitro experiment.

\section{- Conclusion}

Picroside II attenuated testicular I/R injury in rats, mainly through suppressing apoptosis and oxidative stress, and the mechanism was probably through reduction of nitric oxide synthesis.

\section{- Acknowledgement}

To the Department of Urology, Renmin Hospital of Wuhan University.

\section{- References}

1. Pogorelić Z, Mrklić I, Jurić I, Biočić M, Furlan D. Testicular torsion in the inguinal canal in children. J Pediatr Urol. 2013Dec;9(6 Pt A):793-7. doi: 10.1016/j. jpurol.2012.10.013.

2. Pogorelić Z, Mustapić K, Jukić M, Todorić J, Mrklić I, Meštrović J, Jurić I, Furlan D. Management of acute scrotum in children: a 25-year single center experience on 558 pediatric patients. Can J Urol. 2016;23(6):8594-601. doi: 10.1016/s0022-3468(03)00151-9.

3. Sharp VJ, Kieran K, Arlen AM. Testicular torsion: diagnosis, evaluation, and management. Am Fam Physician. 2013;88:835-40. doi: 10.1136/bmj.f7437.

4. Minutoli L, Puzzolo $D$, Rinaldi $M$, Irrera $N$, Marini $H$, Arcoraci V, Bitto A, Crea G, Pisani A, Squadrito F, Trichilo V, Bruschetta D, Micali A, Altavilla D. ROS-mediated NLRP3 inflammasome activation in brain, heart, kidney, and testis ischemia/reperfusion injury. Oxid Med Cell Longev. 2016;2016:1-10. doi: 10.1155/2016/2183026.

5. Altavilla D, Romeo C, Squadrito F, Marini $H$, Morgia G, Antonuccio P, Minutoli L. Molecular pathways involved in the early and late damage induced by testis ischemia: evidence for a rational pharmacological modulation. Curr Med Chem. 2012;19:1219-24. doi: 10.2174/092986712799320538.

6. Turner TT, Bang HJ, Lysiak JL. The molecular pathology of experimental testicular torsion suggests adjunct therapy to surgical repair. J Urol. 2004;172:2574-8. doi: 10.1097/01. ju.0000144203.30718.19.

7. Wang T, Zhai L, Zhang H, Zhao L, Guo Y. Picroside II inhibits the MEK-ERK1/2-COX2 signal pathway to prevent cerebral ischemic injury in rats. J Mol Neurosci. 2015;57:335-51. doi: 10.1111/1440-1681.12445.

8. Li JZ, Yu SY, Mo D, Tang XN, Shao QR. Picroside II inhibits hypoxia/reoxygenation-induced cardiomyocyte apoptosis by ameliorating mitochondrial function through a mechanism involving a decrease in reactive oxygen species production. Int J Mol Med. 2015;35:446-52. doi: 10.3892/ ijmm.2014.2009.

9. Wang $L$, Liu $X H$, Chen $H$, Chen $Z Y$, Weng XD, Qiu T, Liu L. Picroside II protects rat kidney against ischemia/ reperfusion-induced oxidative stress and inflammation by the TLR4/NF-kappaB pathway. Exp Ther Med. 2015;9:1253-8. doi: 10.3892/etm.2015.2225.

10. Wang $Y$, Hong $Y$, Zhang $C$, Shen $Y$, Pan YS, Chen RZ, Zhang Q, Chen $\mathrm{YH}$. Picroside II attenuates hyperhomocysteinemiainduced endothelial injury by reducing inflammation, oxidative stress and cell apoptosis. J Cell Mol Med. 2019:464-75. doi: 10.1111/jcmm.13949.

11. Li S, Wang T, Zhai L, Ge K, Zhao J, Cong W, Guo Y. Picroside II Exerts a Neuroprotective effect by inhibiting mPTP permeability and EndoG Release after cerebral ischemia/reperfusion injury in rats. J Mol Neurosci. 2018;64(1):144-55. doi: 10.1007/s12031-017-1012-z.

12. Bilommi R, Nawas BA, Kusmayadi DD, Diposarosa R, Chairul A, Hernowo BS. The effects of glutathione on malondialdehyde expression and seminiferous tubule damage in experimental testicular torsion-detorsion in Wistar rats. J Pediatr Urol. 2013;9(6):1059-63. doi: 10.1016/j.jpurol.2013.03.008.

13. Johnsen SG. Testicular biopsy score count-a method for registration of spermatogenesis in human testes: normal values and results of 335 hypogonadal males. Hormones. 1970;1:2-25. PMID: 5527187.

14. Yang CM, Lin CC, Hsieh H L. High-glucose-derived oxidative stress-dependent Heme oxygenase-1 expression from astrocytes contributes to the neuronal apoptosis. Mol Neurobiol. 2016;54(1):470-83. doi: 10.1007/s12035-0159666-4.

15. Liu SH, Ma K, Xu XB. A single dose of carbon monoxide intraperitoneal administration protects rat intestine from injury induced by lipopolysaccharide. Cell Stress Chaperones. 2010;15(5):717-27. doi: 10.2307/20799965.

16. Negoescu A, Lorimier P, Labat-Moleur F, Drouet C, Robert C, Guillermet C, Brambilla C, Brambilla E. In situ apoptotic cell labeling by the TUNEL method: Improvement and evaluation on cell preparations. J Histochem Cytochem. 1996;44(9):959-68. doi: 10.1177/44.9.8773561. 
17. Rochette L, Lorin J, Zeller M, Guilland JC, Lorgis L, Cottin Y, Vergely C. Nitric oxide synthase inhibition and oxidative stress in cardiovascular diseases: possible therapeutic targets? Pharmacol Ther. 2013;140(3):239-57. doi: 10.1016/j.pharmthera.2013.07.004.

18. Mogilner JG, Lurie M, Coran AG, Nativ O, Shiloni E, Sukhotnik I. Effect of diclofenac on germ cell apoptosis following testicular ischemia-reperfusion injury in a rat. Pediatr Surg Int. 2006;22:99-105. doi: 10.1007/s00383005-1580-9.

19. MeštrovićJ, Drmić-Hofman I, PogorelićZ, Vilović K, Šupe-Domić D, Šešelja-Perišin A, Capkun V. Beneficial effect of nifedipine on testicular torsion-detorsion injury in rats. Urology. 2014;84(5):1194-8. doi: 10.1016/j.urology.2014.07.022.

20. Ozbek O, Altintas R, Polat A, Vardi N, Parlakpinar $H$, Sagir $M$, Duran ZR, Yildiz A. The protective effect of apocynin on testicular ischemia-reperfusion injury. J Urol. 2015;193(4):1417-22. doi: 10.1016/j.juro.2014.11.086.

21. Şener TE, Yüksel M, Özyılmaz-Yay N, Ercan F, Akbal C, Şimşek $\mathrm{F}$, Şener G. Apocynin attenuates testicular ischemiareperfusion injury in rats. J Pediatr Surg. 2015;50(8):13827. doi: 10.1016/j.jpedsurg.2014.11.033.

22. Meštrović J, Pogorelić Z, Drmić-Hofman I, Vilović K, Todorić $D$, Popović M. Protective effect of urapidil on testicular torsion-detorsion injury in rats. Surg Today. 2017;47(3):16. doi: 10.1007/s00595-016-1388-3.

23. Yurtçu $M$, Abasiyanik $A$, Biçer $S$, Avunduk $M C$. Efficacy of antioxidant treatment in the prevention of testicular atrophy in experimental testicular torsion. J Pediatr Surg. 2009;44(9):1754-8. doi: 10.1016/j. jpedsurg.2008.11.043.

24. Abdel-Gaber SA, Mohammed RK, Refaie MMM. Mechanism mediating the protective effect of diacerein in ischemia-reperfusion-induced testicular injury in rats. Life Sci. 2018;209:57-62. doi: 10.1016/j.Ifs.2018.07.060.

25. Cuzzocrea S, Riley DP, Caputi AP, Salvemini D. Antioxidant therapy: a new pharmacological approach in shock, inflammation, and ischemia/reperfusion injury. Pharmacol Rev. 2001;53(1):135-59. doi: 10.0000/PMID11171943.

26. Zhang Y, Lv Y, Liu YJ, Yang C, Hu HJ, Meng XE, Li MX, Pan SY. Hyperbaric oxygen therapy in rats attenuates ischemiareperfusion testicular injury through blockade of oxidative stress, suppression of inflammation, and reduction of nitric oxide formation. Urology. 2013;82(2):489.e9-489.e15. doi: 10.1016/j.urology.2013.04.016.

27. Lysiak JJ. The role of tumor necrosis factor-alpha and interleukin-1 in the mammalian testis and their involvement in testicular torsion and autoimmune orchitis. Reprod Biol Endocrinol. 2004;2:9. doi: 10.1186/1477-7827-2-9.
28. Gultekin A, Tanriverdi H I, Inan S, Yilmaz O, Gunsar C, Sencan A. The effect of tunica albuginea incision on testicular tissue after detorsion in the experimental model of testicular torsion. Urol J. 2018;15(1):32-9. doi: 10.22037/uj.v0i0.3804.

29. Boettcher $M$, Meier $D$, Jiménez-Alcázar $M$, Eschenburg G, Mietzsch S, Vincent D, Klinke M, Trochimiuk M, Appl B, Tiemann B, Bergholz R, Reinshagen K, Fuchs TA3. Degradation of extracellular DNA by DNase1 significantly reduces testicular damage after testicular torsion in rats. Urology. 2017;109(223):223.e1-7. doi: 10.1016/j. urology.2017.07.031.

30. Kostakis ID, Zavras N, Damaskos C, Sakellariou S, Korkolopoulou P, Misiakos EP, Tsaparas P, Vaos G, and Karatzas T. Erythropoietin and sildenafil protect against ischemia/reperfusion injury following testicular torsion in adult rats. Exp Ther Med. 2017;13(6):3341-7. doi: 10.3892/ etm.2017.4441.

31. Kanter M. Protective effects of melatonin on testicular torsion/detorsion-induced ischemia-reperfusion injury in rats. Exp Mol Pathol. 2010;89(3):314-20. doi: 10.1016/j. yexmp.2010.07.006.

32. Filho DW, Torres MA, Bordin AL, Crezcynski-Pasa TB, Boveris A. Spermatic cord torsion, reactive oxygen and nitrogen species and ischemia-reperfusion injury. Mol Aspects Med. 2004;25(1-2):199-210. doi: 10.1016/j.mam.2004.02.020.

33. Kamalvand G, Pinard G, Ali-Khan Z. Heme-oxygenase-1 response, a marker of oxidative stress, in a mouse model of AA amyloidosis. Amyloid. 2003;10(3):151-9. doi: 10.3109/13506120308998997.

34. Li SW, Fujino M, Takahara T, Li XK. Protective role of heme oxygenase- 1 in fatty liver ischemia-reperfusion injury. Med Mol Morphol. 2019;52(2):61-72. doi: 10.1007/s00795018-0205-z.

35. Kim KA, Kim JY, Lee YA, Song KJ, Min D, Shin MH. NOX1 participates in ROS-dependent cell death of colon epithelial Caco2 cells induced by Entamoeba histolytica. Microbes Infect. 2011;13(12):1052-61. doi: 10.1016/j. micinf.2011.06.001.

36. Erol B, Sari U, Amasyali AS, Ozkanli S, Sogut $S$, Hanci V, Efiloglu O, Danacioglu YO, Engin P, Yencilek F, Atis G, Yildirim A, Alkoc OA, Caskurlu T. Comparison of combined antioxidants and thymoquinone in the prevention of testis ischemia-reperfusion injury. Andrology. 2017;5(1):119-24. doi: 10.1111/andr.12268.

37. Fakouri A, Asghari A, Akbari G, Mortazavi P. Effects of folic acid administration on testicular ischemia/reperfusion injury in rats. Acta Cir Bras. 2017;32(9):755-66. doi: 10.1590/s0102-865020170090000008. 


\section{Correspondence:}

Xiuheng Liu

Zhang Road Wuchang District No. 99

Jiefang Road 238, Wuhan, Hubei, P.R. China

Phone: 027-88041911

drliuxh@hotmail.com

Received: July 16, 2019

Review: Sept 18, 2219

Accepted: Oct 13, 2019
Conflict of interest: none

Financial sources: Application and Basic Research Project of Wuhan City (No. 2018060401011321), Wuhan Morning Light Plan of Youth Science and Technology (No.2017050304010281), Natural Science Foundation of Hubei Province (No. 2016CFB114, 2017CFB181), and Research Project of Wuhan University (No. 2042017kf0097)

${ }^{1}$ Research performed at Central Laboratory, Renmin Hospital of Wuhan University, Wuhan, Hubei, P.R. China. 